$21^{\text {st }}$ European Symposium on Computer Aided Process Engineering - ESCAPE21

E.N. Pistikopoulos, M.C. Georgiadis and A.C. Kokossis (Editors)

(C) 2011 Elsevier B.V. All rights reserved.

\title{
A systematic methodology for the synthesis of unit process chains using Life Cycle Assessment and In- dustrial Ecology Principles
}

\author{
Léda Gerber, Jérôme Mayer, François Maréchal \\ Industrial Energy Systems Laboratory (LENI), Ecole Polytechnique Fédérale de Lau- \\ sanne, CH-1015 Lausanne, Switzerland, leda.gerber@epfl.ch
}

\begin{abstract}
The integration of environmental impacts in the conceptual process design procedure can be achieved by combining Life Cycle Assessment (LCA) tools with flowsheeting software and process integration techniques. However, current LCI databases assume a given unit process chain for each product or waste, and do not allow for the systematic synthesis of the process chain minimizing the environmental impacts of raw materials or auxiliary flows, accounting systematically for the different alternatives and recyclings. This paper presents a methodology for systematically designing process units LCI database for the optimal design of integrated systems with a life cycle perspective.
\end{abstract}

Keywords: process design, process synthesis, optimization, environmental impacts, life cycle assessment, industrial ecology

\section{Introduction}

Integration of environmental impacts in the conceptual process design procedure has gained considerable interest in the last decade. A way of achieving it is to combine Life Cycle Assessment (LCA) tools (ISO (2006a,b)) with flowsheeting software and process integration techniques, which allows for linking the Life Cycle Inventory (LCI) with the process design decisions like the process configuration, scale and operating conditions (Gerber et al. (2010)). To account for the auxiliary materials, the waste generated and the logistics included in the LCA system boundaries, the decision perimeter of the process flowsheet has to be extended. The off-site impacts generated by these flows during their production or treatment is evaluated with the help of a LCI database, and have an important contribution to the total impact, as demonstrated in our previous study. However, for products that can be produced in different ways, current LCI databases like ecoinvent ${ }^{\circledR}$ (Frischknecht et al. (2005)) assume a fixed unit process chain for each product or waste, in which pre-defined fractions of each unit process represent the market share of each option. In consequence, they do not allow for the systematic synthesis of the process chains. Therefore, process design options may be biased due to the choices of the options in the process chains. Moreover, industrial ecology principles, dealing with the closing of material loops by the identification of industrial symbioses is not possible with such databases and with the methodology presented in our previous study, which includes only energy and not mass integration, which would be useful for the systematic identification of recycling possibilities of waste or co-products generated by a process or a range of processes operating at a given location. A tool allowing to use dynamically LCI databases would reveal be useful for process synthesis, industrial parks design, or for allocation of resources and waste at the scale of a territory. Specifically in the field of process design and synthesis, several studies that aim at integrating and minimizing environmental impacts have been already conducted. Hugo and Pistikopoulos (2005) introduce a strategy to syn- 
$21^{\text {st }}$ European Symposium on Computer Aided Process Engineering - ESCAPE21

E.N. Pistikopoulos, M.C. Georgiadis and A.C. Kokossis (Editors)

(C) 2011 Elsevier B.V. All rights reserved.

thesize supply chains, considering both costs and cumulated impacts from life cycle impact assessment in a multi-objective optimization using mixed integer linear programming (MILP) techniques. Guillén-Gosálbez et al. (2008) propose as well a similar approach to directly extract the optimal flowsheet configurations from the process superstructure for the design of chemical processes in the multi-objective optimization, but using mixed integer non-linear programming (MINLP). Extending this approach, Guillén-Gosálbez and Grossman (2010) present a strategy for the multi-objective optimization of chemical supply chains, including uncertainties in the environmental impact model. However, none of these studies considers specifically the LCI database itself as part of the superstructure to be used for unit process chain synthesis. The present study precisely deals with this aspect, and presents a systematic methodology for the synthesis of unit process chains using LCI databases, minimizing the environmental impacts in a life cycle perspective. It accounts simultaneously for both the substitution alternatives and the potential recyclings.

\section{General concept}

In our previous work, we integrated a LCA model in a multi-objective computation framework using a MINLP formulation, as presented in Figure 1, by analogy with the economic performance calculation. However, the presented framework uses a two-step optimization, the first optimization being the process integration part with respect to one objective using a MILP formulation, which is here renamed 'system integration', simultaneously accounting for mass and energy integration. Two-step decomposition is justified since we have a first linear non-differentiable problem with integer variables, and then a nonlinear problem for which we want to perform a multi-objective optimization (Gassner and Maréchal (2009)). Here, we extend the process integration to the overall system integra-

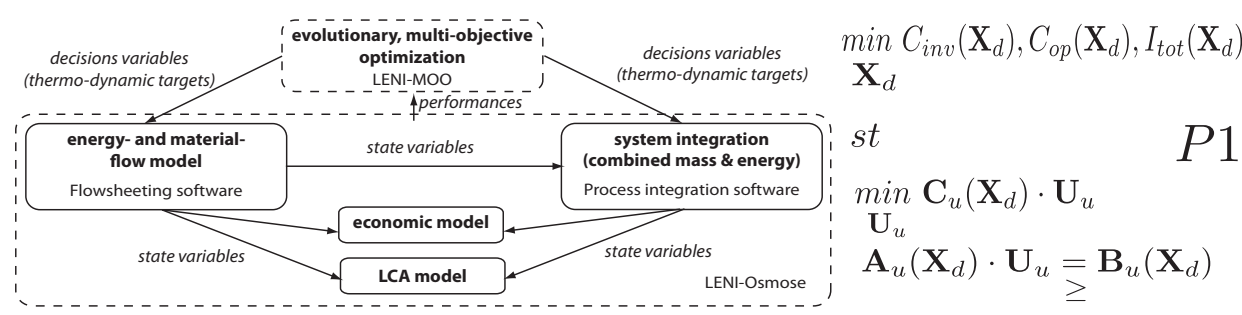

Figure 1: Process system design framework

tion, including the process chains of the auxiliary materials, logistics and resources which are locally necessary to the process. In the present work, we focus on the synthesis of these process chains using LCI data in order to minimize their cost or impact. Therefore, in the costs calculation of the $c_{u}$, we add the cost of the environmental taxes linked to the different impacts, for example the $\mathrm{CO} 2$ tax. The general concept developed for the methodology to account for LCI unit process chain synthesis is described in Figure 2. A distinction is made between the overall system, representing the real system to be considered for calculating the environmental impacts or the total costs, and the action system, where decisions can be effectively taken in order to decrease the impacts or costs. The action system contains a superstructure of unit processes (U), used to synthesize the supply chain necessary to obtain a given amount of final products $(\mathrm{P})$, or to convert available resources (R). A unit process is constituted of a series of sources and sinks (S), representing material and energy flows that are necessary inputs or product outputs. Each unit process generates emissions and extractions (E) to and from the environment, creating impact (I), following the LCA methodology. The process chain is then synthesized by establishing 
$21^{\text {st }}$ European Symposium on Computer Aided Process Engineering - ESCAPE21

E.N. Pistikopoulos, M.C. Georgiadis and A.C. Kokossis (Editors)

(C) 2011 Elsevier B.V. All rights reserved.

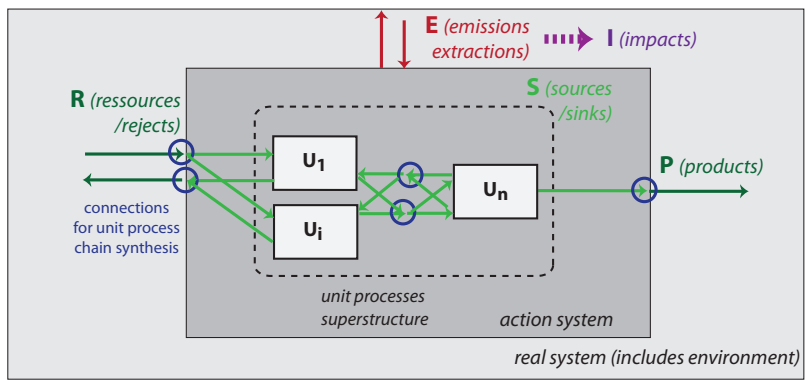

Figure 2: General concept for process chain synthesis from LCI database

matches between sources and sinks, with respect to the goal of the study and the definition of the functional unit (FU), according to the LCA methodology. This FU can be a certain amount of product to be produced, or resources to be converted or waste to be treated. This general concept is used as a basis to disaggregate in a first step the LCI database ecoinvent ${ }^{\circledR}$ in unit processes with associated emissions and extractions, and in a second step to re-synthesize the unit process chain accounting for the mass balance constraints, identifying automatically recyclings and minimizing impacts on the environment or costs.

\section{LCI database disaggregation}

To disaggregate the LCI database ecoinvent ${ }^{\circledR}$, it has to be distinguished between the unit processes of s-type, which are fully included in the superstructure of the action system, having both sources and sinks, and the unit processes of r-type and p-type, which are at the interface of the action system and the environment, r-type unit processes representing a resource delivery, and p-type unit processes representing a product sink. The r-type and p-type units represent the limits of the system under which decisions have to be taken. This is summarized in Figure 3. The level of cut-off at which a unit process becomes a r-
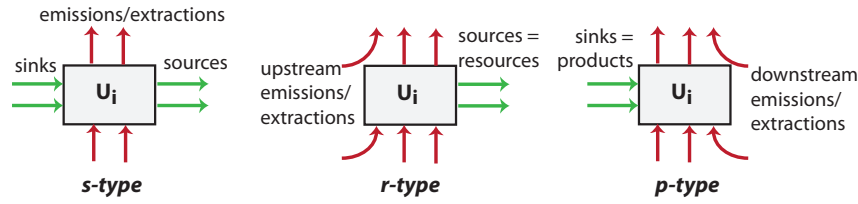

Figure 3: Differences between the s-type, r-type and p-type unit process

or p-type has to be defined considering the level at which no control is possible anymore on the unit processes, representing the average technology or resources available on the market. It is relatively easy to perform this operation with the data from ecoinvent ${ }^{\circledR}$, since the database offers two different formats for the information of the unit processes:

1. UPR format, used for s-type unit processes, which contains the inputs and outputs in terms of material and energy flows, representing the sources and sinks. It contains as well the emissions and extractions generated by the unit process only.

2. Aggregated LCI format, used for $\mathrm{r}$ - and p-type unit processes, containing the cumulative emissions and extractions of the upstream or downstream unit processes.

Once the disaggregation is performed, the different unit processes constitute the superstructure that will be used at next step to re-synthesize the optimal unit process chain. A 
$21^{\text {st }}$ European Symposium on Computer Aided Process Engineering - ESCAPE21

E.N. Pistikopoulos, M.C. Georgiadis and A.C. Kokossis (Editors)

(C) 2011 Elsevier B.V. All rights reserved.

table of correspondence is written for matching sinks and sources ontology.

\section{MILP formulation for unit process chain synthesis}

To synthesize the unit process chain and account for the systematic recylings, we use a MILP model, which is an extension of the methodology and tools presented in Gassner and Maréchal (2009) and Gerber et al. (2010). The constraints are put on the mass balance for each unit process, to ensure that each source for a unit process finds another unit process as a sink, except in the case a certain quantity of a product is wanted:

$$
\begin{aligned}
& \forall s=1 \ldots n_{s}: \sum_{u=1}^{n_{u}} U_{u} \cdot s_{s, u}^{+}\left(x_{d, u}\right)=0, \forall r=1 \ldots n_{r}: \sum_{u=1}^{n_{u}} U_{u} \cdot r_{r, u}^{+}\left(x_{d, u}\right)=R_{r}, \\
& \forall p=1 \ldots n_{p}: \sum_{u=1}^{n_{u}} U_{u} \cdot p_{p, u}^{+}\left(x_{d, u}\right)=P_{p}
\end{aligned}
$$

where $U_{u}$ is the utilization fraction of unit process $u$ of the superstructure for the considered action system, $n$ is the total number of unit processes embedded in the superstructure and $s^{+}$is the quantity of source $s$ consumed by unit process $u$, a negative sign meaning a production. $s^{+}$is function of the decision variables $x_{d}$, involved in the non-linear optimization of the process design method of Figure 1. $r^{+}$is the quantity of resource $r$, and $p^{+}$is the quantity of product $p$. Using this layer approach for each source/sink, product and resource allows to systematically identify the potential recyclings within the action system. The emissions and extractions calculation is as well included in the formulation:

$$
\forall e=1 \ldots n_{e}: \sum_{u=1}^{n_{e}} U_{u} \cdot e_{e, u}^{+}\left(x_{d, u}\right)=E_{e}
$$

where $e_{e, u}^{+}$is the emission or extraction $e$ generated by the unit process $u$, function of the decision variables $x_{d}$, and $E_{e}$ is the total emission or extraction of substance $e$ from the overall system, equivalent to the LCI in the conventional LCA methodology. $s^{+}, r^{+}$, $p^{+}$and $e^{+}$include not only the processed flows, but also the support materials and the equipments. From the emissions and extractions inventory, we can then perform the Life Cycle Impact Assessment (LCIA) and obtain the total impacts of the system:

$$
\forall i=1 \ldots n_{i}: \sum_{e=1}^{n_{e}} f_{e, i} \cdot E_{e}=I_{i}
$$

where $f_{e, i}$ is the weight attributed by the chosen LCIA method to the emission $e$ for the impact $i$, and $I_{i}$ is the impact from the overall system. If the impact assessment method proposes a single score with an additional weighting step, the total impact is written as:

$$
I_{\text {tot }}=\sum_{i=1}^{n} w_{i} \cdot I_{i}\left(U_{u}\right)
$$

where $I_{t o t}$ is the total single score impact and $w_{i}$ is the weight of the impact category $I_{i} . I_{i}$ is function of the decision variables $U_{u}$.

\section{Optimization problem}

Then, the master optimization problem P2 can be written as:

$$
\min C_{i n v}\left(x_{d, u}\right), C_{o p}\left(x_{d, u}\right), I_{t o t}\left(x_{d, u}\right)
$$


$21^{\text {st }}$ European Symposium on Computer Aided Process Engineering - ESCAPE21

E.N. Pistikopoulos, M.C. Georgiadis and A.C. Kokossis (Editors)

(C) 2011 Elsevier B.V. All rights reserved.

which is the non-linear part of the problem solved by a multi-objective evolutionary algorithm, $C_{i n v}$ being the investment cost, $C_{o p}$ the operating cost, $I_{t o t}$ the total environmental impact of the system. It defines the slave subproblem minimizing the total cost:

$$
\min C_{t o t}=C_{i n v}\left(i r\left(x_{d, u}\right), t, U_{u}\right)+t \cdot C_{o p}\left(U_{u}\right)+\sum_{i=1}^{n_{i}} I_{i}\left(U_{u}\right) \cdot c_{e n v}\left(x_{d, u}\right)
$$

where $C_{i n v}$ is the investment cost, accounting for the interest rate $i r$ and the number of years $t$ considered for the lifespan, $C_{o p}$ is the annual operating cost, $c_{e n v}$ is the environmental cost and represent the application of a tax, which can be function of the decision variables $x_{d}$. This is subject to the constraints defined in Equations 1 to 4 .

\section{Conclusions and perspectives}

The presented methodology can be applied to solve a wide range of different problems and provide help in decision-making procedures or in territorial management. For example, it can help to identify the systematic possibilities of industrial ecology at the scale of an industrial park or a territory that satisfy the least cost or the least impact. By accounting for the overall process chain, it can as well be useful to calculate the impact of environmental policies on the different possible conversion chains for a given resource, such as the CO2-tax for example, by calculating its real cost on the overall life cycle.

\section{References}

Frischknecht, R., Jungbluth, N., Althaus, H.-J., Doka, G., Dones, R., Heck, T., Hellweg, S., Hischier, R., Nemecek, T., Rebitzer, G., and Spielmann, M. 2005. The ecoinvent database: Overview and methodological framework. International Journal of Life Cycle Assessment, 10:3-9.

Gassner, M. and Maréchal, F. 2009. Methodology for the optimal thermo-economic, multi-objective design of thermochemical fuel production from biomass. Computers \& Chemical Engineering, 33:769-781.

Gerber, L., Gassner, M., and Maréchal, F. 2010. Systematic Integration of LCA in process systems design: Application to combined fuel and electricity production from lignocellulosic biomass. Computers \& Chemical Engineering, page doi:10.1016/j.compchemeng.2010.11.012.

Guillén-Gosálbez, G., Caballero, J., and Jiménez, L. 2008. Application of Life Cycle Assessment to the Structural Optimization of Process Flowsheets. Industrial and Engineering Chemistry Research, 47:777-789.

Guillén-Gosálbez, G. and Grossman, I. 2010. A global optimization strategy for the environmentally conscious design of chemical supply chains under uncertainty in the damage assessment model. Computers \& Chemical Engineering, 34:42-58.

Hugo, A. and Pistikopoulos, E. 2005. Environmentally conscious long-range planning and design of supply chain networks. Journal of Cleaner Production, 13:1471-1491.

ISO 2006a. Environmental management Đ Life Cycle Assessment Đ Principles and framework. International Standard, ISO 14'040.

ISO 2006b. Environmental management Đ Life Cycle Assessment Đ Requirements and guidelines. International Standard, ISO 14'044. 